\title{
ORIGINAL ARTICLE Polymorphisms of the oxytocin receptor gene and overeating: the intermediary role of endophenotypic risk factors
}

C Davis ${ }^{1,2}, \mathrm{~K} \mathrm{Patte}^{3}, \mathrm{C}$ Zai $^{4}$ and JL Kennedy ${ }^{2,4}$

BACKGROUND/OBJECTIVES: Oxytocin (OXT) is an evolutionarily ancient neuropeptide with strong links to affiliative and prosocial behaviors, and the management of stress. Increases in OXT also tend to decrease food intake, especially of sweet carbohydrates. The social correlates of low OXT levels mesh with the social deficits and stress proneness identified in interpersonal models of overeating, as well as the increased appetite for highly palatable foods typically seen in chronic overeaters. The objectives of this study were to investigate links between polymorphisms of the oxytocin receptor (OXTR) gene and overeating, and to examine OXTR links with relevant endophenotypes of overeating related to reward and stress sensitivity, and to food preferences.

SUBJECT/METHODS: The sample comprised 460 adults between the ages of 25 and 50 years recruited from the community, and representing a broad range of body weights. Overeating, reward and punishment sensitivity, and food preferences, were quantified as composite variables using well-validated questionnaires. In addition, seven single-nucleotide polymorphisms (rs237878, rs237885, rs2268493, rs2268494, rs2254298, rs53576, rs2268498) of the OXTR gene were genotyped.

RESULTS: Analyses identified a four-marker haplotype that was significantly related to food preferences. Individual genotype analyses also found that at least one of the markers was related to each of the phenotypic variables. In addition, an empirically derived structural equation model linking genetic and phenotype variables produced a good fit to the data.

CONCLUSIONS: The results of this preliminary study have demonstrated that OXTR variation is associated with overeating, and with endophenotypic traits such as sweet and fatty food preferences, and reward and punishment sensitivity. In general, the genetic findings also favor the view that overeating may be associated with relatively low basal OXT levels.

Nutrition \& Diabetes (2017) 7, e279; doi:10.1038/nutd.2017.24; published online 22 May 2017

\section{INTRODUCTION}

Oxytocin (OXT) is an evolutionarily ancient neuropeptide, which influences a broad range of affiliative, and related survival behaviors. ${ }^{1}$ As such, OXT is popularly called the 'love hormone, because it aids in the regulation of our sensitivity to reward, including parental attachment, pair bonding and empathy; and it facilitates use of these behaviors to reduce stress. ${ }^{3-5}$ In essence, increases in OXT tend to amplify one's responsiveness to the importance of social cues so that both rewarding and aversive events become more impactful on the individual. ${ }^{6}$ Not surprisingly, the relationship between OXT and social behaviors is moderated by salient environmental factors. ${ }^{2}$ For example, OXT's effects on prosocial behavior will depend on whether the context is affiliation-supportive and whether there are any social barriers to closeness. ${ }^{2}$

\section{OXT and food intake}

In recent years, there has been a flurry of interest in the anorexigenic properties of OXT. ${ }^{7}$ For instance, it is well established that exogenous administration of OXT tends to decrease meal duration and to reduce food intake, especially of sweet carbohydrates. ${ }^{7,8}$ It also appears that OXT selectively inhibits hedonic eating-rather than deprivation-induced eating-by diminishing brain-reward dopaminergic signaling. ${ }^{9}$ Moreover, there seems to be a specific functional relationship between OXT and sugar since an OXT antagonist stimulated sucrose intake at a 10-times lower dose compared with its effect on other carbohydrates such as glucose or on an artificial sweetener (saccharin). ${ }^{10}$ However, recent evidence suggests that OXT is really a 'conditional anorexigen', given that feeding in a social context has been shown to abolish its inhibitory effects on food intake. ${ }^{11}$

Endogenous OXT levels are also relevant to food intake and weight gain in a similar manner. In accord with its role in sugarspecific satiety, mice deficient in OXT also tend to overconsume sucrose solutions, both initially and over a sustained period of time. ${ }^{12}$ Similarly, it has been shown that OXTR-deficient mice tended to develop late-onset obesity. ${ }^{13}$ In addition, serum OXT levels were lower in obese adults and those with type 2 diabetes than in normal-weight controls. ${ }^{14}$ Relatedly, results from a postmortem study of patients with Prader-Willi Syndrome (a disorder characterized by excessive food consumption and weight gain) showed a large reduction of OXT-expressing neurons in the paraventricular nucleus and smaller OXT cell volumes compared with their control counterparts. ${ }^{15}$

To date, studies of OXT signaling in the risk for, and development of, eating disorders are relatively scarce and mostly based on small-sample research. ${ }^{16}$ Of relevance, however, are the findings that chronic sucrose overconsumption tends to downregulate the anorexigenic OXT system in such a way that an elevated food-load 'threshold' is required to induce satiety. ${ }^{17}$ For instance, a recent study found that binge-prone rats consumed $\sim 30 \%$ more sucrose in a non-stressful situation, and $60 \%$ more sucrose in a stressful environment, compared with their non-

\footnotetext{
${ }^{1}$ Kinesiology and Health Sciences, York University, Toronto, ON, Canada; ${ }^{2}$ Department of Psychiatry, University of Toronto, Toronto, ON, Canada; ${ }^{3}$ School of Public Health and Health Systems, University of Waterloo, Waterloo, ON, Canada and ${ }^{4}$ Neurogenetics Department, Centre for Addiction and Mental Health, Toronto, ON, Canada. Correspondence: Dr C Davis, Kinesiology and Health Sciences, York University, 343 Bethune College, 4700 Keele Street, Toronto, ON, M3J 1P3 ON, Canada.

E-mail: cdavis@yorku.ca
}

Received 19 March 2017; accepted 26 March 2017 
binge-prone counterparts-findings that may also be moderated by OXT levels. ${ }^{18}$ Likewise, there is good clinical evidence that chronic overeaters have higher reward responsiveness to palatable food, and tend to experience greater food cravings, compared with their normal-weight counterparts. ${ }^{19}$ Of relevance is the evidence that OXT is a well-established anxiolytic agent that tends to buffer cortisol responses to stress, especially in those with poor emotion-regulation abilities. ${ }^{20}$

\section{Interpersonal theories of overeating}

It is striking that the social deficits associated with relatively low levels of OXT_-such as attachment avoidance, relationship difficulties, parental insensitivity, and stress proneness-show remarkable similarities to the social impairments identified in interpersonal models of overeating. ${ }^{21}$ For instance, several studies have identified poor interpersonal skills, avoidance of emotional expression, interpersonal distrust and a diminished ability to cope with negative feelings across diverse samples of adults with clinically significant overeating. ${ }^{22,23}$ Furthermore, significant links have been established between attachment avoidance and binge eating, ${ }^{24}$ and between attachment insecurity and increased risk for disordered eating in general. ${ }^{25}$ Binge eating in both mothers and fathers has also been associated with poor parent-child interactions during feeding and with the child's emotionalbehavioral problems over time. ${ }^{26}$ Similarly, overeating-especially among those who binge eat-has been associated with an elevated proneness to stress, and with poor emotional regulation. ${ }^{27}$ Interestingly, a large population study recently found that across all forms of disordered eating, patients were more likely to be childless than were their healthy counterparts with an odds ratio of $1.86{ }^{28}$ Such outcomes might have as much to do with relationship and social-bonding deficits as with poor physical reproductive health.

\section{An OXT-related model of overeating}

Based on the body of research reviewed above, it is proposed that OXT may have important implications in the risk for, and development of, clinically significant overeating. Relatively low trait levels of OXT may foster a propensity to overconsume, especially in an environment where highly palatable temptations are ubiquitous, easily available and reasonably inexpensive, and among those with elevated sensitivity to the rewarding properties of such foods. Over time, chronic consumption of a sugar-laden diet may exacerbate the consumption of sweet substances. Relatively low OXT levels are also associated with a myriad of interpersonal deficits, which may contribute to elevated stress, particularly in those who are sensitive to social rejection and anxiousness. Taken together, these factors may foster increases in palatable food consumption as a means of emotional comfort. Such a stress-diathesis model of overeating meshes with the interconnected influences of the OXT system on complex human behaviors and personality traits.

The aim of the current exploratory study was to examine links between markers of the OXTR gene and overeating. We also investigated associations between the OXTR markers and established endophenotypes of overeating such as sensitivity to both punishment and reward, and palatable food preferences. ${ }^{29,30}$ In a relatively large sample of adult men and women, structural equation modeling (SEM) was used to assess simultaneously the relationships proposed in Figure 1.

\section{MATERIALS AND METHODS}

\section{Participants and procedures}

The sample consisted of 460 adults (females $=346$ ) between 24 and 50 years of age. Inclusion criteria were residence in North America for at least 5 years before enrollment in the study, and fluency in written and spoken

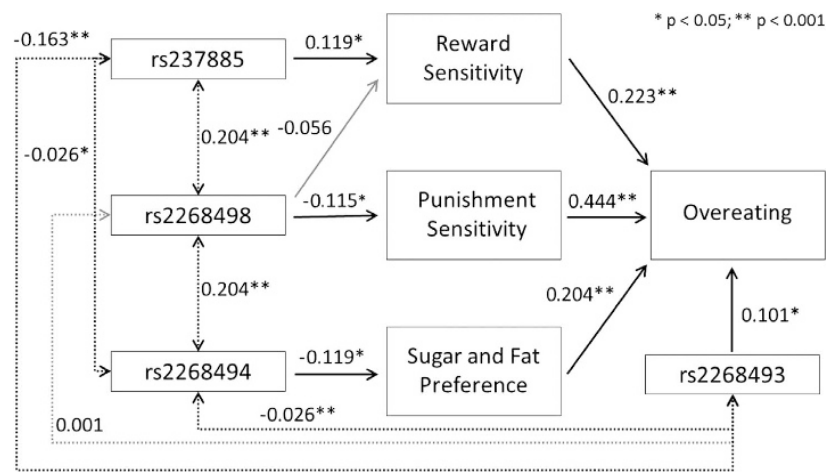

Figure 1. SEM (with standardized regression weights) showing relationships among OXTR polymorphisms, overeating and endophenotypic risk factors for overeating.

English. Women were also required to be premenopausal as indicated by the self-report of a regular menstrual cycle. Exclusion criteria included serious medical illnesses such as cancer or diabetes, or severe physical disabilities such as cerebral palsy. Those with a current axis I diagnosed disorder, with the exception of unipolar depression, were also excluded. In addition, women with a pregnancy in the previous 6 months, or who were lactating, were not included. The majority of the sample was Caucasian (79\%), with $15 \%$ identifying as African descent and the remainder as 'Other'. The sample represented a broad range of body mass index (BMI) values $\left(17.8-75.2 \mathrm{~kg} \mathrm{~m}^{-2}\right)$.

Participants comprised a community-based sample and were recruited from posters, newspaper advertisements and online sites such as Craigslist and Kijiji in a large Canadian city. After a brief telephone screening, an appointment was made for each participant to visit the authors' laboratory at a large clinical and research facility. Each participant was given the questionnaire package to complete. Height and weight were measured with the participant standing in stocking feet and wearing light indoor clothing, and a blood sample was taken at the on-site medical laboratory. The procedures used in this study were approved by the institutional Research Ethics Boards and were carried out in accordance with the Declaration of Helsinki.

\section{Selection of OXTR gene markers}

Seven single-nucleotide polymorphisms (SNPs) in the OXTR gene, localized on 3p25.3, were selected for inclusion in this study based on their reported associations with the salience of, and sensitivity to, both rewarding and aversive social stimuli, and with other aspects of human social behavior. ${ }^{31}$ We also included SNPs with a demonstrated link to eating-related behaviors. $^{32,33}$

For instance, the $\mathrm{G}$ allele at the intron-3 of the rs 53576 marker has been associated with higher reward dependence, increased amygdalar activation during the facial expression face-matching task ${ }^{34}$ higher levels of trust and empathy, ${ }^{35}$ more sensitive parenting ${ }^{36}$ and increased sensitivity to social support. ${ }^{37}$ Additionally, the GG genotype carriers were more likely to report dieting to lose weight and less likely to eat sugary and fatty foods, such as cakes and puddings. ${ }^{38}$

At the intron-3 of the rs 2268493 marker, the T allele has been associated with social impairment, autism spectrum disorders, and attention deficit/ hyperactivity disorder. ${ }^{39-41}$ There is other evidence that this SNP may impact brain-reward circuitry. For instance, the $\Pi \pi$ genotype group displayed decreased activation in mesolimbic regions (including the nucleus accumbens and amygdala) in response to a monetary-rewards task. ${ }^{3}$ The $\mathrm{T}$ allele was also associated with poorer performance on a composite social cognition index. ${ }^{42}$

The A-allele carriers at another intron-3 marker, rs2268494, has been associated with reduced anger responses in a laboratory game focused on interpersonal interactions. ${ }^{43}$ Using a cumulative risk index including the $A A$ genotype of this SNP, another recent study found less empathic concern to their romantic partner's distress and lower social reciprocity in supportgiving interactions. ${ }^{44}$

The $\mathrm{G}$ allele at the intron-3 of the rs2254298 marker has been associated with reduced social anxiety, decreased responsivity to stressors and lower social impairments. ${ }^{45,46}$ Those carrying the $G$ allele also showed lower amygdala response in tasks involving socially relevant face stimuli. ${ }^{47}$ 
In addition, a case-control study of patients with eating disorders found that A-allele carriers had a greater preoccupation with food and weight, and heightened social anxiety. ${ }^{32}$

The $\Pi$ genotype at the intron-3 of the rs 237885 marker has been broadly overrepresented in schizophrenia patients, ${ }^{48}$ and in another study, the $\Pi$ genotype carriers displayed increased callous-unemotional traits compared with their counterparts. ${ }^{49}$ Of relevance, the prepulse inhibition of the startle reflex was significantly lower in the $\Pi$ genotype. ${ }^{50}$ As low prepulse inhibition is associated with depression, these findings mesh with the results of the studies described above.

The T allele of the promoter rs 2268498 marker has been associated with higher scores on a measure of empathy, ${ }^{51}$ and on a measure of socialperception abilities including the strength of social bonding, such as kinship and intimacy. ${ }^{52}$ The $\Pi$ genotype was also associated with the lowest scores on measures of fear and sadness, as well as on negative emotionality. ${ }^{53}$

Finally, the C allele at the $3^{\prime}$ rs 237878 marker has been associated with higher levels of extraversion-a personality construct with strong positive links to reward sensitivity (RS) and sensation seeking-compared with the T allele. ${ }^{54}$

\section{Questionnaire measures}

The three psychological independent variables (reward sensitivity, punishment sensitivity and food-reward preferences), and the dependent variable (overeating), were operationally defined as separate composite variables (using principal components analysis) to reflect the multidimensionality of each construct.

Reward sensitivity. RS comprises three questionnaires, which were designed to assess approach motivation-one of three evolutionarily adaptive brain systems underlying current psychobiological theories of personality. ${ }^{55}$ This neurocircuitry regulates the incentive to obtain rewards as well as the hedonic experience of pleasure derived from these rewards. ${ }^{56}$ The 30-item Barratt Impulsivity Scale identifies facets of impulsiveness such as non-planning, and the tendency to act rashly and to make quick decisions. ${ }^{57}$ The Reward Subscale of the Sensitivity to Punishment and Sensitivity to Reward Questionnaire contains 24 forcedchoice items assessing the respondent's approach responses under various conditions of reward-including tangible rewards such as money and pleasure-inducing substances, as well social rewards such as receiving admiration from others. ${ }^{58}$ And finally, the 13 -item Behavioral Activation Scale comprises three subscales, which collectively assess (i) anticipation of, and positive response to, rewards, (ii) the degree of persistence in achieving rewarding goals, and (iii) the strength of one's desire for these rewards 59

Punishment sensitivity. PS includes three questionnaires, which reflect avoidance motivation-another evolutionarily adaptive brain system regulating the drive to avoid or retreat from situations of threat or punishment, and from other aversive events. ${ }^{60}$ The 35-item Harm Avoidance Scale of the Temperament and Character Inventory reflects the tendency to inhibit behavior in response to aversive or punishing stimuli. ${ }^{61}$ The Punishment Subscale (RS) of the Sensitivity to Punishment and Sensitivity to Reward Questionnaire contains 24 forced-choice items assessing the respondent's avoidance responses in general situations involving worry and the threat of mistreatment, failure and the anticipation of non-reward. ${ }^{58}$ And finally, the 7-item Behavioral Inhibition Scale assesses both fear and anxiety responses to aversive social and environmental stimuli and situations. ${ }^{59}$

Food-reward preferences. Food-reward preferences were assessed by subscales of the well-validated Food Preference Questionnaire. ${ }^{62}$ This questionnaire provides hedonic ratings for 72 common food items arranged according to a 2 (FAT: high vs low) $\times 3$ (OTHER MACRONUTRIENT: high simple sugar, high complex carbohydrate, and high protein) matrix with 12 items in each cell. In the current study, High Sugar and High Fat Foods is reflected by the 12 items from the high fat/high simple sugar cell; High Sugar Foods includes the 12 items from the High Simple Sugar/Low Fat cell and the High Fat Foods is the total (24 items) of the High Fat/High Carbohydrate and High Fat/High Protein subscales. The 72 food items of the Food Preference Questionnaire vary systematically and significantly with respect to their macronutrient content.
Overeating. Overeating comprises three scales representing different aspects of overconsumption of (mostly) highly palatable foods. Binge Eating was assessed by the five-item subscale of the Binge Eating Questionnaire, which includes frequency and severity of symptoms such as loss-of-control overeating, and negative affect following a binge. ${ }^{63}$ The Emotional Eating and Snacking on Sweets subscales (10 and 6 items, respectively) of the Eating Behavior Patterns Questionnaire were also included. ${ }^{64}$ The former assesses eating in response to negative moods and often in the absence of hunger. It also reflects the use of palatable food to comfort a distressed state. The latter describes an eating pattern characterized by the frequent consumption of sugary snacks throughout the day, and sometimes as a replacement for regular meals.

\section{Genotyping methods}

Genomic DNA was purified from blood lymphocytes using the high-salt method as described previously. ${ }^{65}$ Genotyping was conducted on OpenArray plates (Life Technologies, Applied Biosystems Inc., Foster City, CA, USA) using a PCR-based method in the QuantStudio $12 \mathrm{~K}$ Flex Real-Time PCR System. We randomly regenotyped $10 \%$ of the samples for quality control purposes.

For the genetic analyses, departure from Hardy-Weinberg equilibrium was tested for each marker, and linkage disequilibrium (LD) values between markers were determined in Haploview version 4.2 (Broad Institute of MIT and Harvard, Cambridge, MA, USA). ${ }^{66}$ Single-marker analyses were conducted using SPSS (IBM Analytics), whereas haplotype analyses were performed with minimum haplotype frequency threshold of 0.05 in UNPHASED version 3.1.7. ${ }^{67}$

\section{Statistical analyses}

The statistical analyses were conducted in two stages. Initially, four oneway analysis of variance procedures were conducted for each SNP, with genotype as the independent variable and RS, PS, food preferences, and overeating as separate dependent variables. These preliminary analyses identified relevant SNPs to include in the SEM illustrated in Figure 1. SEM procedures enable multiple regression equations to be tested simultaneously, which allows for a comprehensive model of the relationships between OXTR markers and endophenotypes associated with overeating. All parameters were determined a priori to testing. A model was tested in which RS, PS, and food preference each predicted overeating, with the SNPs added as predictors of the appropriate variables according to the significant effects identified in the preliminary analyses. The four factor scores were used instead of latent variables to avoid issues of collinearity in testing the SEM model, and covariances were added among the four OXTR SNPs given they were all markers on the same gene.

SEM was performed using SPSS AMOS 24. Missing data were handled using the AMOS estimate means and intercepts function, which applies maximum-likelihood methods. ${ }^{68}$ The $x^{2}$ test and a number of 'approximate' or 'global' fit tests were calculated. The $X^{2}$ analysis is known as the 'exactfit' test in SEM, because a nonsignificant value requires the model-implied population covariance matrix to have zero discrepancies from the actual sample observed covariance matrix.

\section{RESULTS}

\section{Composite variables}

Principal component analysis (SPSS version 23) was used to create the four composite variables, as described in the Methods section. Total scores, not individual items, were entered into the principal component analyses. Each analysis extracted only one component with an eigenvalue $>1$. Table 1 presents the component matrix for each composite variable showing factor loadings, and the percentage of variance accounted for by the extracted component. In all subsequent analyses, the derived factor scores were used to reflect the composite variables.

\section{Descriptive statistics}

Prior to the analyses, all the composite-variable factor scores were screened for normality. None deviated significantly as indicated by skewness values $< \pm 1$. Table 2 presents means and s.d. for the latent variable factor scores, and for age and $\mathrm{BMI}$, reported 
separately for female and male participants. Group differences were assessed by a one-factor multivariate analysis of variance. Results indicated -in accord with previous research - that females reported a greater tendency to overeat, ${ }^{69,70}$ and a more pronounced sensitivity to punishment, ${ }_{1}^{71}$ whereas males demonstrated a great sensitivity to reward. ${ }^{71,72}$

Genotype frequencies and endophenotype analyses

Table 3 lists the genotype frequencies for the seven OXTR SNPS used in the following analyses. None of the genotypes for the seven OXTR SNPs deviated significantly from Hardy-Weinberg equilibrium $(P>0.05)$. Owing to the low frequency of the minor allele for the rs2268493, rs2268494 and rs2254298 SNPs, the homozygous minor-allele group was combined with the heterozygous group to form a binary genotype variable. Additionally, and in light of arguments which favor the reduction of type II errors (that is, the probability of not rejecting the null hypothesis when it is false) in exploratory and preliminary research, ${ }^{73}$ we did not control for multiple testing in the genotype analyses.

There were no group effects for rs53576, rs2254298 and rs237878. However, the remaining four SNPs demonstrated significant genotype differences on at least one endophenotypic

\begin{tabular}{|c|c|c|}
\hline Latent variable & Factor loadings & $\begin{array}{l}\% \text { Explained } \\
\text { variance }\end{array}$ \\
\hline Reward sensitivity $(n=424)$ & & 57.0 \\
\hline 1. IMP & 0.65 & \\
\hline 2. SPSRQ & 0.86 & \\
\hline 3. BAS & 0.74 & \\
\hline Punishment sensitivity $(n=423)$ & & 79.4 \\
\hline 1. Harm Avoidance (TPQ) & 0.90 & \\
\hline 2. SPSRQ & 0.90 & \\
\hline 3. BIS & 0.87 & \\
\hline Food-reward preferences $(n=432)$ & & 87.32 \\
\hline 1. High Sugar Foods & 0.91 & \\
\hline 2. High Fat Foods & 0.95 & \\
\hline $\begin{array}{l}\text { 3. High Sugar and High Fat } \\
\text { Foods }\end{array}$ & 0.94 & \\
\hline Overeating $(n=427)$ & & 70.0 \\
\hline 1. Binge eating & 0.84 & \\
\hline 2. Emotional eating & 0.89 & \\
\hline 3. Snacking on sweets & 0.76 & \\
\hline
\end{tabular}

variable. Findings for the SNPs with significant effects are summarized in Table 4. For rs2268493, the homozygous T group reported greater overeating than the group with at least one copy of the $C$ allele. With respect to rs2268494, the group with at least one copy of the A allele reported a stronger preference for sweet and fatty foods compared with those with the homozygous $T$ genotype.

For rs2268498 there were main effects for both RS and PS with the homozygous $C$ genotype reporting higher sensitivity on both measures. Post hoc comparisons using the least significant difference test indicated that the CC group was higher than both the $C T$ and $\Pi$ groups ( $P=0.020$ and 0.009 , respectively) on PS, whereas the CC group was only higher than the CT group regarding the RS variable $(P=0.015)$.

There was also a rs227885 main effect for RS indicating higher scores among the $G$ homozygous group. Least significant difference post hoc comparisons demonstrated this group had greater scores than both the $\Pi$ and the TG groups $(P=0.012$ and 0.019 , respectively).

Structural equation modeling

Although the current model did produce a significant $X^{2}$ (32.145, d.f. $=14, P=0.004)$, this result is unsurprising given the sample size. $^{74}$ Consequently, it is conventional to examine indices other than the exact-fit $x^{2}$ to determine the quality of the fit of

Table 3. Genotypes frequencies for the seven OXTR SNPs included in the analyses

\begin{tabular}{lcccc}
\hline rs53576 & $A A$ & $A G$ & $G G$ & Total \\
Frequency & 43 & 213 & 201 & 457 \\
\hline rs2268493 & $C C$ & $C T$ & $\pi$ & \\
Frequency & 26 & 169 & 261 & 456 \\
\hline rs2268494 & $A A$ & $A T$ & $\pi$ & \\
Frequency & 5 & 59 & 393 & 457 \\
\hline rs2268498 & $C C$ & $C T$ & $\pi$ & \\
Frequency & 75 & 237 & 144 & 456 \\
\hline rs237878 & $C C$ & $C T$ & $\pi$ & \\
Frequency & 54 & 184 & 189 & 427 \\
\hline rs2254298 & $A A$ & $A G$ & $G G$ & \\
Frequency & 9 & 121 & 325 & 455 \\
\hline rs237885 & $\pi$ & $T G$ & $G G$ & \\
Frequency & 108 & 210 & 123 & 441 \\
\hline Abbreviations: & OXTR, & oxytocin & receptor; & SNP, single-nucleotide \\
polymorphism. & & & & \\
\hline
\end{tabular}

Table 2. Means and s.d. for all quantitative variables included in the study, listed separately for male and female participants

\begin{tabular}{|c|c|c|c|c|c|c|}
\hline Variable & $\mathrm{N}$ & Means (s.d.) & $\mathrm{N}$ & Means (s.d.) & $\mathrm{F}$ & P-value \\
\hline Age & 346 & $33.4(6.6)$ & 115 & $34.3(7.0)$ & 1.92 & 0.166 \\
\hline Reward-Sensitivity Factor Score & 318 & $-0.06(0.97)$ & 106 & $0.18(1.1)$ & 4.88 & 0.028 \\
\hline Punishment-Sensitivity Factor Score & 317 & $0.09(0.98)$ & 106 & $-0.27(1.0)$ & 10.68 & 0.001 \\
\hline
\end{tabular}




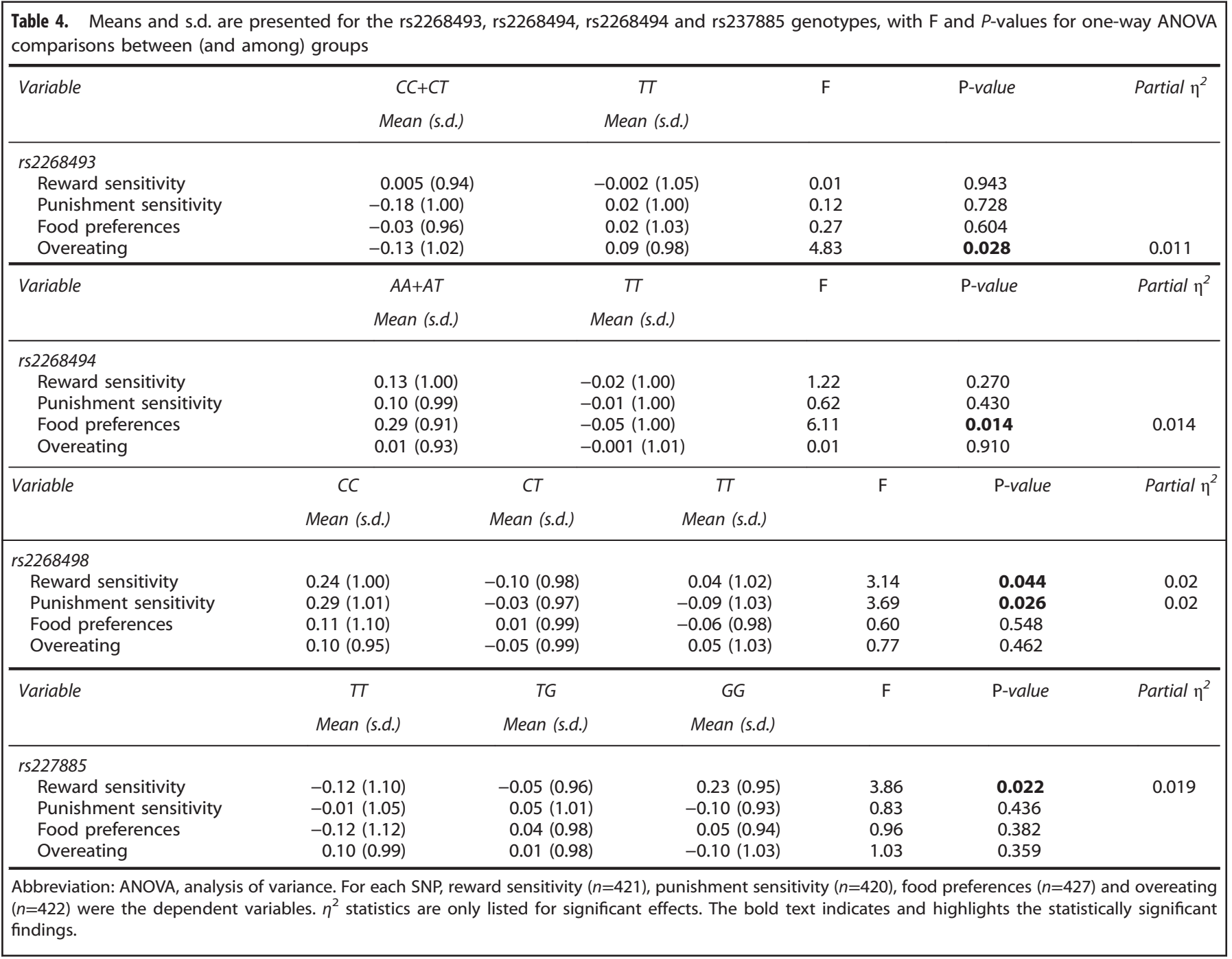

an SEM model. Following is a summary of these statistical indicators.

The relative $x^{2}$ (CMIN/d.f.) measure divides the $x^{2}$ statistic by its degrees of freedom. A value below 3 is deemed an acceptable value for a well-fitting model, ${ }^{74}$ and the current model produced a relative $x^{2}$ of 2.296 . The root mean square error of approximation is the most popular measure of goodness-of-fit, with an acceptable fit indicated by values below 0.06 and lower and upper confidence interval limits below 0.05 and 0.08 , respectively. ${ }^{75}$ The model had a root mean square error of approximation of $0.051(0.028,0.074)$. The Tucker-Lewis index, comparative fit index, and normed fit index should be at least 0.90 , with a well-fitting model scoring 0.95 or greater. ${ }^{75}$ The model resulted in Tucker-Lewis index, comparative fit index, and normed fit index values of $0.870,0.949$ and 0.918 , consecutively.

Overall, and according to well-established statistical criteria, the model was therefore a good fit to the data. AMOS produces estimates of $R^{2}$ (squared multiple correlations). For overeating, the $R^{2}$ value is 0.298 ( 0.301 when controlling for sex in the model). All parameter estimates were in the expected direction and statistically significant, with the exception of the effect of the rs2268498 SNP on RS $(P=0.257)$ and the covariance between the rs2268493 and rs2268498 $(P=0.966)$. See Figure 1 for the standardized regression weights. (Note 1: In light of the significant male-female differences in the mean RS, PS and overeating variables, the SEM was rerun adding paths between sex and these three variables. This change made virtually no difference to the fit of the model as seen by the fit indices reported below. Significant pathways resulted from sex to PS $(-0.152$; $P=0001)$, to $\operatorname{RS}(0.106, P=0.028)$ and to the overeating $(-0.087$, $P=0.036)$ variable. The model fit indices with sex added to the model are: $x^{2}=2.778, P<0.0001, C M I N=49.996$, Tucker-Lewis index $=0.794$, comparative fit index $=0.917$, normed fit index $=$ 0.884 , root mean square error of approximation $=0.060(0.040$ 0.079 ). As negligible differences resulted in the pathway estimates when controlling for sex, it was decided to leave sex out of the model since the fit indices are somewhat better without sex in the model).

Haplotype analyses

Linkage disequilibrium analysis showed that the rs237885, rs2268493, rs2268494 and rs2254298 SNPs formed one haplotype block, and rs53576 and rs2268498 formed another, as seen in Figure 2. Analysis of the four-SNP haplotypes indicated that the G-T-A-G haplotype was associated with higher scores on the food preferences composite variable (haplotype window $P=0.0055$; haplotype frequency $=6.06 \%$; estimated additive value $=0.74 ; 95 \%$; confidence interval: $0.32-1.16$; haplotypespecific $P=0.00046$ ). (Note 2 : Results in the subsample of 


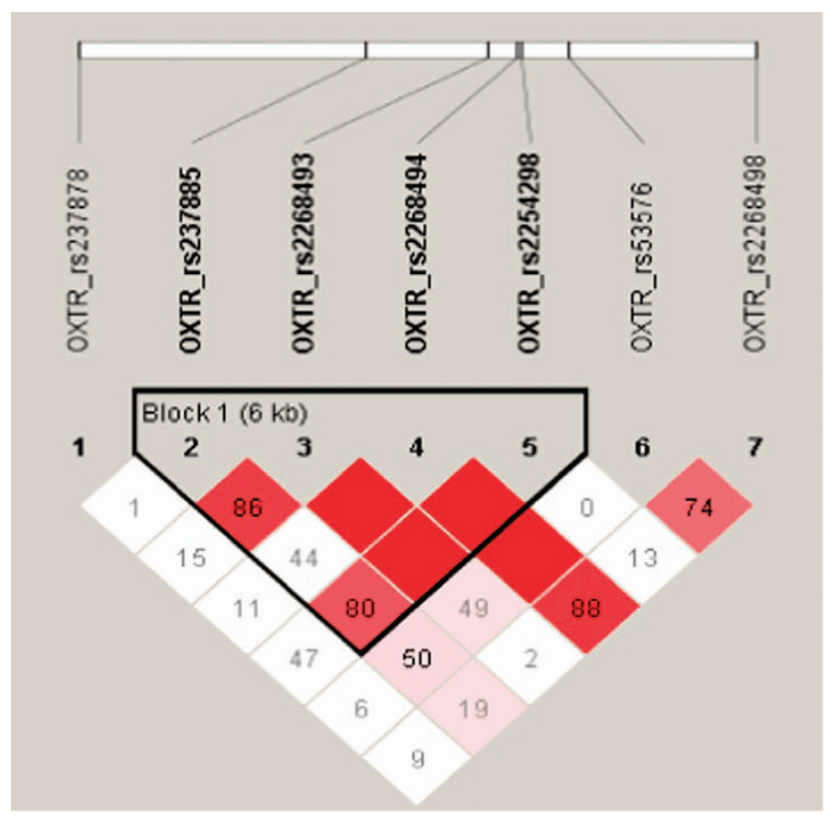

Figure 2. LD plot for the OXTR gene SNPs in our sample of Caucasian participants. The values indicate the pairwise LD $\left(D^{\prime}\right)$, and the intensity of the color scheme is based on $D^{\prime}$. The LD block was defined by an $R^{2}$ threshold of 0.7 on solid spine.

Caucasian participants were similar, with the $\mathrm{G}-\mathrm{T}-\mathrm{A}-\mathrm{G}$ haplotype being associated with higher scores on the food preference composite score (haplotype window $P=0.011$; haplotype frequency $=7.56 \%$; estimated additive value $=0.64 ; 95 \%$ confidence interval: 0.23-1.06; haplotype-specific $P=0.00196)$.

\section{DISCUSSION}

It is well established that large individual differences exist both in basal OXT levels and in the reactivity of the OXT system and the abundance of its receptors-factors that influence responsiveness to social stimuli and that aid in the regulation of food intake. ${ }^{76}$ Generally speaking, a robust OXT system is more resilient and receptive to social reward, and better able to ameliorate anxiety and reduce stress. ${ }^{76}$ These are all characteristics that tend to be diminished in many individuals with chronic overeating. ${ }^{77}$

To the best of our knowledge, this is the first study to examine genetic markers of the OXT system from a broad 'OXT-deficiency' perspective in relation to overeating, and endophenotypes of overeating. SEM was used to assess multiple simultaneous relationships in a theoretical model proposing the influence of psychobehavioral traits on overeating, and their underlying biological links as indicated by relevant OXTR SNPs. Results indicated that our model was a good fit to the data. All specified paths were statistically significant in the predicted direction except the path between rs2268498 and RS, which did not survive the $P$-value imposed by SEM to account for multiple simultaneous tests. Independent and additive associations, in the positive direction, were found for RS, PS and sugar/fat food preferences on overeating $\left(R^{2}=30 \%\right)$. To date, only a handful of studies has examined the simultaneous relationships between RS and PS and eating-related problems, and are in accord with the current findings. ${ }^{78,79}$ Previous evidence also indicates a clear relationship between overeating and elevated BMI, and palatable food preferences. $^{80,19}$

\section{OXT associations}

The significant association between the G-T-A-G haplotype and high sugar/fat food preferences is supportive of the OXTdeficiency viewpoint in the context of overeating. In previous research, the rs237885 G allele was associated with the highest levels of prepulse inhibition, low depressive symptoms ${ }^{50}$ and lower unemotional traits. ${ }^{49}$ The rs2268493 T allele and the rs2268494 A allele have each been associated with poorer social cognition, ${ }^{39-42}$ and less emphatic concern. ${ }^{44}$ Similarly, the GG genotype of rs2254298 has also been associated with lower empathic concern and social reciprocity, and lower salivary OXT levels. ${ }^{44,81}$ Empathy is a key component of prosocial-emotional development with heritability estimates above $50 \%{ }^{82}$ In summary, our haplotype findings suggest that reduced OXT signaling, which is related to social deficits and to lower social responding, may also influence sweet and fatty food preferences. Such a link meshes with the association between low OXT levels and increased sucrose intake. ${ }^{10}$

Single-marker analysis found that four of the seven OXTR SNPs included were associated with at least one of the compositedependent variables. In accord with the haplotype analysis, the A allele carriers at rs2268494 reported higher sweet and fatty food preferences than those with $\Pi$. Relatedly, the $\Pi$ genotype at rs2268493 reported greater overeating compared with the combined CC+CT group. In addition, the GG genotype of the rs237885 marker was related to higher RS compared with the other genotype groups. Finally, the homozygous $C$ group for rs2268498 reported significantly higher RS and PS scores.

It is important to note, in relation to OXT markers, that while there is relative consistency across studies concerning a marker's association with a particular trait or characteristic, the 'risk' allele may differ across samples. That is, the direction of the effect is sometimes inconstant. Such findings support the social salience theory of OXT whereby the effects of OXT are nuanced by stable characteristics of the individual and the environmental context, ${ }^{2}$ so that OXT 'does not cast a rose-colored hue for all'2 (p. 105). The role of other individual differences is therefore important when interpreting the role of OXT on psychobehavioral outcomes, ${ }^{83}$ and an important step forward for future researchers in this area of investigation.

In conclusion, this study presents an innovative psychogenetic risk model of overeating and its relevant endophenotypic correlates. As such, it has several strengths including a sound model based on theoretically and empirically supported relationships. Nevertheless, it is important to also address some limitations in this research. Importantly, replication is essential in all SNP-association studies to strengthen confidence in the current findings. As this was the first study to examine relationships between overeating and its endophenotypes, and markers of the OXTR gene, we hope that other investigators will attempt to confirm our findings in future research. Relatedly, it is necessary to assess findings from Caucasian-specific single-SNP analysis in larger independent samples. As mentioned in the Note 2 above, however, the direction of the current findings were not changed, and remained significant, when the haplotype analysis was carried out in the Caucasian-only sample.

\section{CONFLICT OF INTEREST}

The authors declare no conflict of interest.

\section{ACKNOWLEDGEMENTS}

The phenotype data were funded by a grant (MOP-84257) to the first author (PI) and the last author from the Canadian Institute of Health Research. Genotyping of the OXTR markers was funded by a grant to the first author from Shire Pharmaceuticals. 


\section{REFERENCES}

1 Crespi BJ. Oxytocin, testosterone, and social cognition. Biol Rev Camb Philos Soc 2016; 91: 390-408.

2 Bartz JA. Oxytocin and the pharmacological dissection of affiliation. Curr Dir Psychol Sci 2016; 25: 104-110.

3 Damiano CR, Aloi J, Dunlop K, Burrus CJ, Mosner MG, Kozink RV et al. Association between oxytocin receptor (OXTR) gene and mesolimbic responses to reward. Mol Autism 2014; 5: 7.

4 Feldman R, Zagoory-Sharon O, Weisman O, Schneiderman I, Gordon I, Maoz R et al. Sensitive parenting is associated with plasma oxytocin and polymorphisms in the OXTR and CD38 genes. Biol Psychiatry 2012; 72: 175-181.

5 Loth E, Poline J-B, Thyreau B, Jia T, Tao C, Lourdusamy A et al. Oxytocin receptor genotype modulates ventral striatal activity to social cues and response to stressful life events. Biol Psychiatry 2014; 76: 367-376.

6 McQuaid RJ, McInnis OA, Matheson K, Anisman H. Oxytocin and social sensitivity: gene polymorphisms in relation to depressive symptoms and social ideation. Front Hum Neurosci 2016; 10: 358; article 358.

7 Olszewski PK, Klockers A, Levine AS. Oxytocin: A Conditional Anorexigen whose Effects on Appetite Depend on the Physiological, Behavioural and Social Contexts. J Neuroendocrinol 2016; 28; doi: 10.1111/jne12376.

8 Sabatier N, Leng G, Menzies J. Oxytocin, feeding, and satiety. Front Endocrinol 2013; 4: 35; article 35.

9 Ott V, Finlayson G, Lehnert H, Heitmann B, Heinrichs M, Born J et al. Oxytocin reduces reward-driven food intake in humans. Diabetes 2013; 62: 3418-3425.

10 Herisson FM, Brooks LL, Waas JR, Levine AS, Olszewski PK. Functional relationship between oxytocin and appetite for carbohydrates versus saccharin. Neuroreport 2014; 25: 909-914.

11 Olszewski PK, Allen K, Levine AS. Effect of oxytocin receptor blockade on appetite for sugar is modified by social context. Appetite 2015; 86: 81-87.

12 Amico JA, Vollmer RR, Cai H-M, Miedler JA, Rinaman L. Enhanced initial and sustained intake of sucrose solution in mice with an oxytocin gene deletion. $A m \mathrm{~J}$ Physiol Regul Integr Comp Physiol 2005; 289: R1798-R1806.

13 Takayangi Y, Kasahara Y, Onaka T, Takahashi N, Kawada T, Nishimori K. Oxytocin receptor-deficient mice developed late-onset obesity. NeuroReport 2008; 19: 951-955.

14 Qian WY, Zhu TY, Tang BQ, Yu SQ, Hu H, Sun WJ et al. Decreased circulating levels of oxytocin in obesity and newly diagnosed Type II diabetes patients. J Clin Endocrinol Metab 2014; 99: 4683-4689.

15 Swaab DF, Purba JS, Hofman MA. Alterations in the hypothalamic paraventricular nucleus and its oxytocin neurons (putative satiety cells) in Prader-Willi syndrome: a study of five cases. J Clin Endocrinol Metabol 1995; 80: 573-579.

16 Romano A, Tempesta B, Micioni Di Bonaventura MV, Gaetani S. From autism to eating disorders and more: the role of oxytocin in neuropsychiatric disorders. Front Neurosci 2016; 9: 497.

17 Mitra A, Gosnell BA, Schioth HB, Grace MK, Klockars A, Olszewski PK et al. Chronic sugar intake dampens feeding-related activity of neurons synthesizing a satiety mediator, oxytocin. Peptides 2010; 31: 1346-1352.

18 Calvez J, de Ávila C, Matte LO, Guèvremont G, Gundlach Al, Timofeeva E. Role of relaxin-3/RXFP3 system in stress-induced binge-like eating in female rats. Neuropharmacology 2016; 102: 207-215.

19 Davis C, Patte K, Levitan RD, Reid C, Tweed S, Curtis C. From motivation to behaviour: a model of reward sensitivity, overeating, and food preferences in the risk profile for obesity. Appetite 2007; 48: 12-19.

20 Quirin M, Kuhl J, Dusing R. Oxytocin buffers cortisol responses to stress in individuals with impaired emotion regulation abilities. Psychoneuroendocrinology 2011; 36: 898-904.

21 Arcelus J, Haslam M, Farrow C, Meyer C. The role of interpersonal functioning in the maintenance of eating psychopathology: a systematic review and testable model. Clin Psychol Rev 2013; 33: 156-167.

22 Berger SS, Elliott C, Ranzenhofer LM, Shomaker LB, Hannallah L, Field SE et al. Interpersonal problem areas and alexithymia in adolescent girls with loss of control eating. Compr Psychiatry 2014; 55: 170-178.

23 Ivanova IV, Tasca GA, Proulx G, Bissada H. Does the interpersonal model apply across eating? A structural equation modeling approach. Compr Psychiatry 2015; 63: 80-87.

24 Shakory S, Van Exan J, Mills JS, Sockalingam S, Keating L, Taube-Shiff M. Binge eating in bariatric surgery candidates: the role of insecure attachment and emotion regulation. Appetite 2015; 91: 69-75.

25 Tasca GA, Balfour L. Attachment and eating disorders: a review of current research. Int J Eat Disord 2014; 47: 710-717.

26 Cimino S, Cerniglia L, Porreca A, Simonelli A, Ronconi L, Ballarotto G. Mothers and fathers with binge eating disorder and their 18-36 months old children: a longitudinal study on parent-infant interactions and offspring's emotionalbehavioral profiles. Front Psychol 2016; 7: 580.
27 Berg KC, Crosby RD, Cao L, Peterson CB, Engel SG, Mitchell JE et al. Facets of negative affect prior to and following binge-only, purge-only, and binge/ purge events in women with bulimia nervosa. J Abnorm Psychol 2013; 122: 111-118.

28 Linna MS, Raevuori A, Haukka J, Suvisaari JM, Suokas JT, Gissler M. Reproductive health outcomes in eating disorders. Int J Eating Disord 2013; 46: 826-833.

29 Wu MD, Brockmeyer T, Hartmann M, Skunde M, Herzog W, Friederich H-C. Reward-related decision making in eating and weight disorders: A systematic review and meta-analysis of the evidence from neuropsychological studies. Neurosci Biobehav Rev 2016; 61: 177-196.

30 Vainik U, Dube L, Lu J, Fellows LK. Personality and situation predictors of consistent eating patterns. PLoS One 2015; 10: e0144134.

31 Feldman R, Monakhov M, Pratt M, Ebstein RP. Oxytocin Pathway Genes: Evolutionary Ancient System Impacting on Human Affiliation, Sociality, and Psychopathology. Biol Psychiatry 2016; 79: 174-184.

32 Acevedo SF, Valencia C, Lutter M, McAdams CJ. Severity of eating disorder symptoms related to oxytocin receptor polymorphisms in anorexia nervosa. Psychiatry Res 2015; 228: 641-648.

$33 \mathrm{Kim}$ YR, Kim JH, Kim CH, Shin JG, Treasure J. Association between the oxytocin receptor gene polymorphism (rs53576) and bulimia nervosa. Eur Eat Disord Rev 2015; 23: 171-178.

34 Tost $H$, Kolachana B, Hakimi S, Lemaitre $H$, Verchinski BA, Mattay VS et al. A common allele in the oxytocin receptor gene (OXTR) impacts prosocial temperament and human hypothalamic-limbic structure and function. Proc Natl Acad Sci USA 2010; 107: 13936-13941.

35 Smith KE, Porges EC, Norman GJ, Connelly JJ, Decety J. Oxytocin receptor gene variation predicts empathic concern and autonomic arousal while perceiving harm to others. Soc Neurosci 2014; 9: 1-9.

36 Bakermans-Kranenburg MJ, ljzendoom MH. Oxytocin receptor (OXTR) and serotonin transporter (5-HTT) genes associated with observed parenting. Soc Cogn Affect Neurosci 2008; 3: 128-134.

37 Kanthak MK, Chen FS, Kumstra R, Hill LK, Thayer JF, Heinrichs M. Oxytocin receptor gene polymorphism modulates the effects of social support on heart rate variability. Biol Psychol 2016; 117: 43-49.

38 Connelly JJ, Golding J, Gregory SP, Ring SM, Davis JM, Davey G et al. Personality, behavior and environmental features associated with OXTR genetic variants in British mothers. PLoS One 2014; 9: e90465.

39 Campbell DB, Datta D, Jones ST, Batey Lee E, Sutcliffe JS, Hammock EA et al. Associations of oxytocin receptor (OXTR) gene variants with multiple phenotype domains of autism spectrum disorder. J Neurodev Disord 2011; 3: 101-112.

40 Lerer E, Lerer E, Levi S, Salomon S, Darvasi A, Yirmiya N et al. Association between the oxytocin receptor (OXTR) gene and autism: relationship to Vineland Adaptive Behavior Scales and cognition. Mol Psychiatry 2007; 13: 980-988.

41 Ayaz AB, Karkucak M, Ayaz M, Gokoe S, Kayan E, Goler EE et al. Oxytocin system social function impacts in children with attention-deficit/hyperactivity disorder. Am J Med Genet B Neuropsychiatr Genet 2015; 168: 609-616.

42 Davis MC, Horan WP, Numi EL, Rizzo S, Li W, Sugar CA et al. Associations between oxytocin receptor genotypes and social cognitive performance in individuals with schizophrenia. Schizophr Res 2014; 159: 353-357.

43 Tabak BA, McCullough ME, Carver CS, Pedersen EJ, Cuccaro MI. Variation in oxytocin receptor gene (OXTR) polymorphisms is associated with emotional and behavioral reactions to betrayal. Soc Cogn Affect Neurosci 2014; 9: 810-816.

44 Schneiderman I, Kanat-Maymon Y, Ebstein RP, Feldman R. Cumulative risk on the oxytocin receptor gene (OXTR) underpins empathic communication difficulties at the first stage of romantic love. Soc Cogn Affect Neurosci 2014; 9: 1524-1529.

45 Chen FS, Johnson SC. An oxytocin receptor gene variant predicts attachment anxiety in females and autism-spectrum traits in males. Soc Psychol Pers Sci 2012; 3: 93-99.

46 Parker KJ, Garner JP, Libove RA, Hyde SA, Hornbeak KB, Carson DS et al. Plasma oxytocin concentrations and OXTR polymorphisms predict social impairments in children with and without autism spectrum disorder. Proc Natl Acad Sci USA 2014; 111: $12258-12263$.

47 Marusak HA, Furman DJ, Kuruvadi N, Shattuck DW, Joshi SH, Joshi AA et al. Amygdala responses to salient social cues vary with oxytocin receptor genotype in youth. Neuropsychologia 2015; 79: 1-9.

48 Montag C, Brockmann E-M, Bayerl M, Rujescu D, Muller DJ, Gallinat J. Oxytocin and oxytocin receptor gene polymorphisms and risk for schizophrenia: a casecontrol study. World J Biol Psychiatry 2013; 14: 500-508.

49 Beitchman JH, Zai CC, Muir K, Berall L, Nowrouzi B, Choi E et al. Childhood aggression, callous-unemotional traits and oxytocin genes. Eur Child Adolesc Psychiatry 2012; 21: 125-132.

50 Comasco E, Gulinello M, Hellgren C, Skalkidou A, Sylven S, Sundstrom-Poromaa I. Sleep duration, depression, and oxytocinergic genotype influence prepulse inhibition of the startle reflex in postpartum women. Eu Neuropsychopharmacol 2016; 26: 767-776. 
51 Christ CC, Carlo G, Stoltenberg SF. Oxytocin Receptor (OXTR) Single Nucleotide Polymorphisms Indirectly Predict Prosocial Behavior Through Perspective Taking and Empathic Concern. J Pers 2016; 84: 204-213.

52 Melchers M, Montag C, Felten A, Reuter M. The oxytocin receptor gene and social perception. Soc Neurosci 2015; 10: 345-353.

53 Montag C, Fiebach CJ, Kirsch P, Reuter M. Interaction of 5-HTTLPR and a variation on the oxytocin receptor gene influences negative emotionality. Biol Psychiatry 2011; 69: 601-603.

54 Haram M, Tesli M, Dieset I, Steen NE, Rossberg Jl, Djurovic S et al. An attempt to identify single nucleotide polymorphisms contributing to possible relationships between personality traits and oxytocin-related genes. Neuropsychobiology 2014; 69: 25-30.

55 Krupic D, Gracanin A, Corr PJ. The evolution of the Behavioural Approach System (BAS): cooperative and competitive resource acquisition strategies. Pers Indiv Diff 2016; 94: 223-227.

56 Berridge KC, Kringelbach ML. Neuroscience of affect: brain mechanisms of pleasure and displeasure. Curr Opin Neurobiol 2013; 23: 294-303.

57 Patton JH, Stanford MS, Barratt ES. Factor structure of the Barratt Impulsivity Scale. J Clin Psychol 1995; 51: 768-774.

58 Torrubia R, Avila C, Molto J, Caseras X. The Sensitivity to Punishment and Sensitivity to Reward Questionnaire (SPSRQ) as a measure of Gray's anxiety and impulsivity dimensions. Pers Indiv Diff 2002; 31: 837-862.

59 Carver CS, White TL. Behavioral inhibition, behavioral activation, and affective responses to impending reward and punishment: the BS/BAS Scales. J Pers Soc Psychol 1994; 67: 319-333.

60 Hahn T, Dressler T, Plichta MM, Ehlis AC, Ernst LH, Markulin F et al. Functional amygdala-hippocampus connectivity during anticipation of aversive events is associated with Gray's trait 'Sensitivity to Punishment'. Biol Psychiatry 2010; 68: 459-464.

61 Cloninger CR, Przybeck TR, Svrakic DM, Wetzel RD. The Temperament and Character Inventory (TCI): A Guide to its Development and Use. Center for Psychology and Personality, Washington University: St Louis, MO, USA, 1994.

62 Geiselman JP, Anderson AM, Dowdy ML, West DB, Redman SM, Smith SR. Reliability and validity of a macronutrient self-selection paradigm and a food preference questionnaire. Physiol Behav 1998; 63: 919-928.

63 Halmi KA, Falk JR, Schwartz E. Binge-eating and vomiting: a survey of a college population. Psychol Med 1981; 11: 697-706.

64 Schlundt DG, Hargreaves MK, Buchowski MS. The Eating Behavior Questionnaire predicts dietary fat intake in African American women. J Am Diet Assoc 2003; 103: 338-345.

65 Lahiri DK, Nurnberger JI Jr. A rapid non-enzymatic method for the preparation of HMW DNA from blood for RFLP studies. Nucleic Acids Res 1991; 19: 5444.

66 Barrett JC, Fry B, Maller J, Daly MJ. Haploview: analysis and visualization of LD and haplotype maps. Bioinformatics 2005; 21: 263-265.

67 Dudbridge F. Likelihood-based association analysis for nuclear families and unrelated subjects with missing genotype data. Hum Hered 2008; 66: 87-98.

68 Allison PD. Missing data techniques for structural equation modeling. J Abnorm Psychol 2003; 112: 545-557.

69 Camilleri GM, Mejean C, Kesse-Guyot E, Andreeva VA, Bellisle F, Hereberg S et al. The associations between emotional eating and consumption of energy-dense snack foods are modified by sex and depressive symptomatology. J Nutr 2014; 144: 1264-1273.
70 Jaaskelanen A, Nevanpera N, Remes J, Rahkonen F, Jarvelin M-R. Stress-related eating, obesity and associated behavioural traits in adolescents: a prospective population-based cohort study. BMC Public Health 2014; 14: 321.

71 Lombardo MV, Ashwin E, Auyeung B, Chakrabarti B, Lai M-C, Taylor K et al. Fetal programming effects of testosterone on the reward system and behavioral approach tendencies in humans. Biol Psychiatry 2012; 72: 839-847.

72 Cross CP, Copping LT, Campbell A. Sex differences in impulsivity: a meta-analysis. Psychol Bull 2011; 137: 97-130.

73 Davis C, Gaito J. Multiple comparison procedures within experimental research. Can Psychol 1984; 25: 1-13.

74 lacobucci D. Structural equations modeling: fit indices, sample size, and advanced topics. J Consum Psychol 2010; 20: 90-98.

$75 \mathrm{Hu}$ LT, Bentler PM. Cutoff criteria for fit indexes in covariance structure analysis: Conventional criteria versus new alternatives. Struct Equation Model 1999; 6: 1-55.

76 Buisman-Pijlman FTA, Sumracki NM, Gordon JJ, Hull PR, Carter CS, Tops M. Individual differences underlying susceptibility to addiction: role for the endogenous oxytocin system. Pharmacol Biochem Behav 2014; 119: 22-38.

77 Calvez J, Timofeeva E. Behavioral and hormonal responses to stress in binge-like eating prone female rats. Physiol Behav 2016; 157: 28-38.

78 Loxton NJ, Dawe S. How do dysfunctional eating and hazardous drinking women perform on behavioural measures of reward and punishment sensitivity? Pers Indiv Diff 2007; 42: 1163-1172.

79 Matton A, Goossens L, Braet C, Vervaet M. Punishment and reward sensitivity: are naturally occurring clusters in these traits related to eating and weight problems in adolescents? Eur Eat Disord Rev 2013; 21: 184-194.

80 Bojanowska E, Ciosek J. Can we selectively reduce appetite for energy-dense foods? An overview of pharmacological strategies for modification of food preference behavior. Curr Neuropharmacol 2016; 14: 118-142.

81 Apter-Levy Y, Feldman M, Vakart A, Ebstein RP, Feldman R. Impact of maternal depression across the first 6 years of life on the child's mental health, social engagement, and empathy: The moderating role of oxytocin. Am J Psychiatry 2013; 170: 1161-1168.

82 Melchers M, Montag C, Reuter M, Spinath FM, Hahn E. How heritable is empathy? Differential effects of measurement and subcomponents. Mot Emot 2016; 40: 720-730

83 McDonald NM, Baker JK, Messinger DS. Oxytocin and parent-child interaction in the development of empathy among children at risk for autism. Dev Psychol 2016; 52: 735-745.

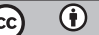

This work is licensed under a Creative Commons Attribution 4.0 International License. The images or other third party material in this article are included in the article's Creative Commons license, unless indicated otherwise in the credit line; if the material is not included under the Creative Commons license, users will need to obtain permission from the license holder to reproduce the material. To view a copy of this license, visit http://creativecommons.org/licenses/ by/4.0/

(c) The Author(s) 2017 